\section{FGFR allosteric inhibition}

\section{By Lauren Martz, Staff Writer}

Sanofi and collaborators at the Flanders Institute for Biotechnology at Catholic University Leuven have serendipitously discovered an allosteric inhibitor of the cancer target fibroblast growth factor receptor. The team hopes the allosteric mechanism will lead to improved safety over molecules that bind the receptor's active site and that the identification of this molecule will pave the way for designing other allosteric inhibitors-a process that has proven challenging. ${ }^{1,2}$

Fibroblast growth factor receptor (FGFR) is often mutated and overexpressed in cancers, in which it plays a role in angiogenesis. The FGFR family is made up of 4 different receptors, all of which have different splice variants, and 22 different ligands.

Although some FGFR signaling pathways contribute to cancer pathogenesis, others
"The work has a lot of possibilities for different therapeutic areas, and there are a lot of receptor tyrosine kinases implicated in cancer that could benefit from allosteric inhibition."

-W. Mike Kavanaugh, Five Prime Therapeutics Inc.
The team for the first paper was led by Jean-Marc Herbert, head of the Early-to-Candidate Unit at Sanofi, and Peter Carmeliet, professor of medicine and director of the Vesalius Research Center at VIB-Catholic University Leuven. The papers also included researchers from the Yale School of Medicine, The University of Tokyo, the University of Liege, Goethe University Frankfurt, the Spanish National Cancer Research Center (CNIO) and Monash University.

The researchers did not set out to find an allosteric inhibitor but rather set up a high throughput screen designed to discover orthosteric FGFR inhibitors. In that screen, the team identified a compound that had low binding affinity for the FGFR ligand-binding site but nonetheless had high activity against FGFR ligand-driven endothelial cell proliferation.

This inhibitory pattern was not typical of orthosteric inhibition and uggested that the compound could act as an allosteric inhibitor.

To confirm that SSR inhibits FGFR allosterically, the group mapped its binding site and showed that SSR binds to the D3 extracellular domain of the receptor but not to the FGFR ligand-binding site located on the D2 domain.

In various cell lines, SSR inhibited FGFR. The compound had no effect on cancer cells lacking FGFR and did not alter the responses of other receptor tyrosine kinases to ligands.

Oral SSR plus a mAb targeting mouse Vegf receptor 2 (Kdr/Flk-1; Vegfr-2) also inhibited and delayed growth and decreased invasiveness and metastasis of Vegfr-resistant tumors in mice compared with either treatment alone. These findings suggest that FGFR allosteric are important for normal physiological functioning. Nonspecific inhibition of FGFR signaling can cause serious side effects such as hyperphosphatemia.

Thus, inhibiting the target can cause both on- and off-target toxicities. In addition, FGFR inhibitors often have cross-reactivity with other receptor tyrosine kinases, which can lead to problems with blood pressure.

An even bigger challenge is that the FGFR family is large and structurally redundant, making it hard to target specific components. Indeed, competitive inhibitors of the ligand-binding sites may nonspecifically block binding of all ligands.

Sanofi and the Flanders Institute for Biotechnology (VIB) have found a potential solution by identifying an allosteric inhibitor of FGFR.

Allosteric inhibitors are difficult to design because it is often unclear which types of binding and which binding locations will noncompetitively alter the structure of the receptor. However, such compounds have the potential to improve specificity by targeting variable regions of closely related receptors, decreasing required drug doses and bypassing resistance to existing drugs.

In one study, a group led by Sanofi and VIB generated mouse disease data on an allosteric FGFR inhibitor, SSR128129E (SSR). In another paper, many of the same researchers studied the molecular structure of SSR and its mechanism of FGFR inhibition in more depth.

Both papers were published in Cancer Cell. inhibition could help overcome resistance to VEGFR inhibitors.

In a mouse model for arthritis, oral SSR decreased clinical severity, slowed disease progression and decreased the number of limbs affected by the disease compared with vehicle control. SSR blocked angiogenesis in the limbs, which decreased infiltration of inflammatory lymphocytes. These findings suggest the antiangiogenic effects of SSR could be applied to diseases other than cancer.

In the second paper, the goal was to confirm the allosteric binding mode of SSR. This paper also included researchers from the University of Cambridge and the Hungarian Academy of Sciences.

The team ruled out competitive binding with FGFR ligands, impaired dimerization of the receptor or ligands and general tyrosine kinase inhibition as SSR's mechanism. They also found that SSR binding to FGFR prevented internalization of the receptor into the cell, which could help inhibit downstream receptor signaling.

In binding experiments, the group showed that SSR bound all constructs that contained the D2 or D3 extracellular domains of FGFR, confirming that the compound interacted with the membrane receptor's extracellular domain.

2D NMR and crystallography studies pinpointed an extracellular binding site of an SSR-related compound on the FGFR D2 domain that was distinct from the known ligand or co-receptor binding sites. NMR and crystallography studies could not be used to identify the interaction 
of SSR with the D3 extracellular domain due to the dynamic, unfolded and disordered nature of the region.

Instead, Fourier transform infrared (FTIR) spectroscopy and in silico modeling were used to assess the effect of SSR on the D3 domain. These showed that the molecule induced a structural change in the domain that produced a new hydrophobic pocket on the receptor that may help alter ligand binding and receptor signaling.

"It has been very difficult to identify such molecules, and this sort of information could lead to direct screening programs for these types of compounds," said W. Mike Kavanaugh, CSO and SVP of research at Five Prime Therapeutics Inc. "The work has a lot of possibilities for different therapeutic areas, and there are a lot of receptor tyrosine kinases implicated in cancer that could benefit from allosteric inhibition."

Carmeliet added, "It will be useful to explore whether other receptor tyrosine kinases also have allosteric sites that can be targeted by small molecules. Sites exhibiting conformational flexibility are particularly interesting. This would require, however, a different and more demanding screening strategy than the traditional screening for orthosteric inhibitors, and it is here that the challenges lie."

\section{Allosteric advantage}

Carmeliet told SciBX that "Sanofi has

"What is especially important
is that this group has
identified a completely new
way to block the receptor....
We must give credit to this
group, which has clearly done
something very difficult."
$\quad$-Neil Thompson,
Astex Pharmaceuticals Inc.

Carmeliet thinks SSR's allosteric mechanism could circumvent some of the safety issues associated with FGFR inhibition.

"Orthosteric inhibitors block ligands that activate the receptors. If tumors upregulate the ligands, then higher doses of orthosteric inhibitors must be used to competitively antagonize the ligands," he said. "Such high doses of the inhibitors can cause toxic side effects for healthy tissues, as is the case with orthosteric VEGF inhibitors. By contrast, an allosteric inhibitor acts independently of the ligand concentration and is therefore safer to use as it cannot be overdosed."

Regardless of how a molecule blocks FGFR signaling, toxicity could still be an issue because it is not yet clear which downstream FGFR signaling pathways are selectively blocked by SSR.

Kevin Baker, VP of preclinical development at Five Prime, said SSR is still a general inhibitor of FGFR and still blocks most downstream signaling of the receptors, so there is the potential for related side effects such as elevated blood phosphate.

Carmeliet countered that SSR does not block all downstream FGFR signaling, as orthosteric inhibitors normally do, but acknowledged that the specific effects still need to be characterized.

Five Prime and GlaxoSmithKline plc are developing 3052230, an FGF ligand trap that selectively blocks cancer-associated FGF ligands while only weakly binding hormone- associated FGFs, which can result in toxicity.

Carmeliet told SciBX that the team is trying to identify new allosteric inhibitors for other receptor tyrosine kinases.

Sanofi and VIB have joint patents covering their screening method, and Sanofi has patented SSR and related analogs. The IP is not available for licensing

Martz, L. SciBX 6(17); doi:10.1038/scibx.2013.406

Published online May 2, 2013

\section{REFERENCES}

1. Bono, F. et al. Cancer Cell; published online April 15, 2013; doi:10.1016/j.ccr.2013.02.019

Contact: Peter Carmeliet, Catholic University Leuven, Leuven, Belgium

e-mail: peter.carmeliet@vib-kuleuven.be

2. Herbert, C. et al. Cancer Cell; published online April 15, 2013; doi:10.1016/j.ccr.2013.02.018

Contact: Françoise Bono, Sanofi Research and Development, Toulouse, France

e-mail: francoise.bono@sanofi-aventis.com

Contact: Harald Schwalbe, Goethe University Frankfurt, Frankfurt, Germany

e-mail: schwalbe@nmr.uni-frankfurt.de

Contact: Francesco Luigi Gervasio, Spanish National Cancer Research Center (CNIO), Madrid, Spain

e-mail: flgervasio@cnio.es

\section{COMPANIES AND INSTITUTIONS MENTIONED}

Astex Pharmaceuticals Inc. (NASDAQ:ASTX), Dublin, Calif.

Catholic University Leuven, Leuven, Belgium

Five Prime Therapeutics Inc., South San Francisco, Calif.

Flanders Institute for Biotechnology, Ghent, Belgium

GlaxoSmithKline plc (LSE:GSK; NYSE:GSK), London, U.K.

Goethe University Frankfurt, Frankfurt, Germany 


\section{ANALYSIS}

Hungarian Academy of Sciences, Budapest, Hungary Johnson \& Johnson (NYSE:JNJ), New Brunswick, N.J. Monash University, Melbourne, Victoria, Australia Sanofi (Euronext:SAN; NYSE:SNY), Paris, France Spanish National Cancer Research Center, Madrid, Spain University of Cambridge, Cambridge, U.K.

\section{TARGETS \& MECHANISMS}

University of Liege, Liege, Belgium

The University of Texas MD Anderson Cancer Center, Houston, Texas

The University of Tokyo, Tokyo, Japan

Yale School of Medicine, New Haven, Conn. 